\title{
Energy functional and fixed points of a neural network
}

\author{
Leonid B. Litinsky \\ Russia, 142092, Troitsk, Moscow Region, \\ Institute for High Pressure Physics Russian Academy of Sciences \\ fax: /095/-334-0012, e-mail: litin@ns.hppi.troitsk.ru
}

\begin{abstract}
A dynamic system, which is used in the neural network theory, Ising spin glasses and factor analysis, has been investigated. The properties of the connection matrix, which guarantee the coincidence of the set of the fixed points of the dynamic system with the set of local minima of the energy functional, have been determined. The influence of the connection matrix diagonal elements on the structure of the fixed points set has been investigated.
\end{abstract}

\section{Introduction}

We define a neural network as a dynamic system of $n$ spin variables (spins) which can take one of two values:

$$
\sigma_{i}=\{ \pm 1\}, \quad i=1,2, \ldots, n .
$$

The spins are connected by a symmetric connection matrix $J=\left(J_{i j}\right)$ :

$$
J_{i j}=J_{j i}, \quad i, j=1,2, \ldots, n .
$$

The local potential

$$
h_{i}(t)=\sum_{j=1}^{n} J_{i j} \sigma_{j}(t)
$$

with which the network acts on $\operatorname{spin} i$, determines solely the value of spin $i$ at time $t+1$ :

$$
\sigma_{i}(t+1)=\left\{\begin{array}{rlr}
\sigma_{i}(t), & \text { if } & h_{i}(t) \sigma_{i}(t) \geq 0 \\
-\sigma_{i}(t), & \text { if } & h_{i}(t) \sigma_{i}(t)<0
\end{array}\right.
$$

The state of the network as a whole is described by a configuration vector $\vec{\sigma}$, whose coordinates are given by Eq.(1). In what follows, Greek letters will be used to designate configuration vectors.

We want to investigate the set of the so called fixed points of the network, i.e. such states $\vec{\sigma}^{*}$, that for all coordinates $\sigma_{i}^{*}$ have:

$$
\sigma_{i}^{*} h_{i} \geq 0, \quad i=1,2, \ldots, n
$$


Besides the neural network theory, the mathematical model (1)-(3) is also used in the factor alalysis [1] and in the Ising spin glass theory [2]. The neural network theory makes use of a physical concept of the energy of the state $\vec{\sigma}$, which is defined as

$$
E(\vec{\sigma})=-\frac{1}{n} \sum_{i=1}^{n} \sigma_{i} h_{i}=-\frac{1}{n} \sum_{i, j=1}^{n} J_{i j} \sigma_{i} \sigma_{j} .
$$

It is very important, both from the physical point of view and the ability of the network to have content-addressable memory [2], that the energy of the state would be a decreasing function on every step of the network evolution. And, moreover, the fixed points must be the local minima of the energy functional (5).

In the second section, we obtain the conditions under which a connection matrix $J$ guarantees the fulfillment of the above mentioned requirements. It has been found that the Hebb connection matrix as well as the connection matrices which are used in physical problems possess the necessary property. But this is not the case for the projection matrix [3]. As a result, a network with such a connection matrix has a set of fixed points which is wider than the set of the local minima of the energy functional (5). In the third section, we show how the situation for a network with a projection matrix can be improved.

Notations. In what follows, a network with a connection matrix $J$ is called a $J$ network. We denote by $F P(J)$ the set of all fixed points of the $J$-network. A configuration vector which is a fixed point of a network will have a superscript "*":

$$
\vec{\sigma}^{*} \in F P(J) .
$$

We denote by $L M(J)$ the set of the local minima of the energy functional (5). To examine the local minima of the functional (5), we introduce a topology on the set of configuration vectors: the set of $n$ configuration vectors $\vec{\sigma}^{(l)}$ which are the nearest to the vector $\vec{\sigma}$ in the sense of the Hamming distance will be called a vicinity of the state $\vec{\sigma}$ :

$$
\vec{\sigma}^{(l)}=\left(\sigma_{1}, \sigma_{2}, \ldots,-\sigma_{l}, \ldots, \sigma_{n}\right), \quad l=1,2, \ldots, n .
$$

In other words, the state $\vec{\sigma}^{(l)}$ from the vicinity of the state $\vec{\sigma}$ differs from the latter by the opposite value of the $l$ th spin only,

$$
\vec{\sigma} \in L M(J) \Leftrightarrow E(\vec{\sigma}) \leq E\left(\vec{\sigma}^{(l)}\right), \quad l=1,2, \ldots, n .
$$

Finally, a matrix with zero diagonal elements will be marked by the superscript " 0 ":

$$
J^{(0)} \Leftrightarrow J_{i i}=0, \quad i=1,2, \ldots, n .
$$

\section{On the role of the diagonal elements}

\section{Theorem 1}

1. The set of the local minima of the energy functional (5) does not depend on the value of the diagonal elements of the connection matrix:

$$
L M(J)=L M(J+A),
$$


where

$$
A=\operatorname{diag}\left\{a_{11}, a_{22}, \ldots, a_{n n}\right\}
$$

and all the elements $a_{i i}$ are arbitrary real numbers.

2. For a connection matrix $J^{(0)}$ with zero diagonal elements, the set of the fixed points coincides with the set of the local minima of the energy functional

$$
F P\left(J^{(0)}\right)=L M\left(J^{(0)}\right) .
$$

3. Let all the elements $a_{i i}$ of the diagonal matrix (6) be positive, then

$$
F P\left(J^{(0)}+A\right) \supseteq F P\left(J^{(0)}\right) \supseteq F P\left(J^{(0)}-A\right) .
$$

The proof of Theorem 1: Let's write the energy of the state $\vec{\sigma}$, extracting the contribution of the $l$ th spin. Up to the positive factor we obtain:

$$
E(\vec{\sigma}) \propto-\sum_{i, j \neq l} J_{i j} \sigma_{i} \sigma_{j}+J_{l l}-2 \sigma_{l} h_{l} .
$$

The state $\vec{\sigma}$ will be the local minimum of the energy functional if and only if the system of the inequalities $(10)$ is fulfilled for all states $\vec{\sigma}^{(l)}$ from the vicinity of the state $\vec{\sigma}$ :

$$
E\left(\vec{\sigma}^{(l)}\right)-E(\vec{\sigma}) \propto \sigma_{l} h_{l}-J_{l l}=\sigma_{l} \sum_{j \neq l} J_{l j} \sigma_{j} \geq 0, \quad l=1,2, \ldots, n .
$$

It is evident that the inequalities (10) do not depend on the values of the diagonal elements. The conditions of their fulfillment are defined by the off-diagonal part of the matrix $J$. By this the first item of the Theorem is proved. Moreover, it follows from the inequalities (10) that for nonnegative $J_{l l}$ any local minimum of the energy functional is also a fixed point of the network:

$$
L M(J) \subseteq F P(J) \text { when } J_{l l} \geq 0, \quad l=1,2, \ldots, n .
$$

Then, let a state $\vec{\sigma}^{*}$ be a fixed point of the network: $\sigma_{l}^{*} h_{l} \geq 0, \quad l=1,2, \ldots, n$. With the help of Eq.(9) we obtain:

$$
E\left(\vec{\sigma}^{(l)}\right)-E\left(\vec{\sigma}^{*}\right) \propto \sigma_{l}^{*} h_{l}-J_{l l}\left\{\begin{array}{rlll}
\geq & 0, & \text { if } & J_{l l}=0 \\
? & 0, & \text { if } & J_{l l}>0 \\
> & 0, & \text { if } & J_{l l}<0
\end{array}\right.
$$

In other words, for nonpositive $J_{l l}$ any fixed point of the network is a local minimum of the energy functional:

$$
L M(J) \supseteq F P(J) \text { when } J_{l l} \leq 0, \quad l=1,2, \ldots, n .
$$

Combined with the proved first item of the Theorem, Eqs. (11) and (12) justify the correctness of Eqs. (7) and (8). Thus, the proof of the Theorem is finished.

In fact, to some extent the Theorem 1 permits regulating the set of the fixed points of the network. Let's explain this statement. If the matrix $J^{(0)}$ is transformed by the diagonal matrix $A, \quad J(A)=J^{(0)}+A$, then in accordance with the Theorem 1 , the set of the local minima of the energy functional is not changed. But this transformation affects the set of the fixed points of the $J(A)$-network. Indeed, if all the matrix elements $a_{i i}$ are 
positive, the set of the $J(A)$-network fixed points extends as compared with $F P\left(J^{(0)}\right)$. The last is true due to the appearance of the new fixed points which are not the local minima. If, on the contrary, all the matrix elements $a_{i i}$ are negative, the set of the fixed points of the $J(A)$-network narrows as compared with $F P\left(J^{(0)}\right)$ : some states, remaining, as they were, the local minima of the energy functional, cease to be the fixed points.

The last statement allows to suggest a simple method for the elimination of the unnecessary fixed points of the network. Let's formulate it in the form of a theorem.

\section{Theorem 2}

Let the fixed points of a $J^{(0)}$-network be numbered in such a way that

$$
E\left(\vec{\sigma}^{(1)} \leq E\left(\vec{\sigma}^{(2)} \leq \ldots \leq E\left(\vec{\sigma}^{(k)}<E\left(\vec{\sigma}^{(k+1)} \leq \ldots\right.\right.\right.\right.
$$

(to simplify the writing, here we omit the superscript "*" in the notations of the fixed points). Let $A$ be a diagonal matrix whose elements are defined by the equalities

$$
a_{i i}=\min _{l=1, k} \sigma_{i}^{(l)} h_{i}\left(\vec{\sigma}^{(l)}\right), \quad i=1,2, \ldots n,
$$

where $h_{i}(\vec{\sigma})$ is the potential (2) which acts on the $i$ th spin in the $J^{(0)}$-network. Then

$$
F P\left(J^{(0)}-A\right)=\left\{\vec{\sigma}^{(1)}, \vec{\sigma}^{(2)}, \ldots, \vec{\sigma}^{(k)}\right\} .
$$

The proof of Theorem 2: Since all $a_{i i}$ from Eq. (13) are positive, the set of the $\left(J^{(0)}-A\right)$-network fixed points, due to Theorem 1, can be only narrower in comparison with the set $J^{(0)}$-network fixed points. And from the definition (4) of a fixed point, it follows that the state $\vec{\sigma}^{(l)}$ will be a $\left(J^{(0)}-A\right)$-network fixed point if and only if the system of the inequalities

$$
\sigma_{i}^{(l)} h_{i}\left(\vec{\sigma}^{(l)}\right)-a_{i i} \geq 0, \quad i=1,2, \ldots, n
$$

is fulfilled. When the definition (13) is taken into account, it is evident that for any fixed point $\vec{\sigma}^{(l)}$ with the $l \leq k$ the system of the inequalities (14) is fulfilled. Consequently, the first $k$ states of the $\vec{\sigma}^{(l)}$ are the $\left(J^{(0)}-A\right)$-network fixed points. On the other hand, by proceeding from Eq.(5) for the energy and taking into account that the $J^{(0)}$-network fixed points are strictly ordered with respect to the energy increase, it is easy to see that at least for one of the coordinates of the state $\vec{\sigma}^{(l)}$ with $l>k$, the inequalities (14) are not fulfilled. Consequently, the states $\vec{\sigma}^{(l)}$ with $l>k$ are not the $\left(J^{(0)}-A\right)$-network fixed points.

Remark. From Eq.(9) it can be easily shown that even under the sequential dynamics the evolution of a network with a connection matrix whose diagonal elements are negative can be accompanied by the energy increase. As a result, even under the sequential dynamics limit cycles can be formed for such a network! From this point of view, the connection matrices with negative diagonal elements are absolutely nonphysical. But Theorem 2 gives a simple and effective method to eliminate high energy fixed points. In

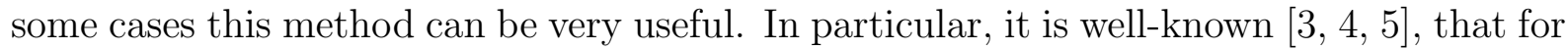
a network with the projection connection matrix the energies of the spurious fixed points are larger then the energies of the memorized patterns. Consequently all such spurious fixed points can be easily eliminated with the help of Theorem 2 . 


\section{Projection connection matrix}

1). Let

$$
\vec{\xi}^{(l)}=\left(\xi_{1}^{(l)}, \xi_{2}^{(l)}, \ldots, \xi_{n}^{(l)}\right), \quad l=1,2, \ldots, p,
$$

be $p$ preassigned configuration vectors which we would like to have as a network fixed points (such vectors are usually called the memorized patterns). It is known [3], that it can be easily done if a matrix $P$ of orthogonal projection onto the linear subspace $\Lambda_{\left.<\vec{\xi}^{(1)}, \vec{\xi}^{(2)}, \ldots, \vec{\xi}^{(p)}\right\rangle}$, spanned by the $p$ memorized patterns $\vec{\xi}^{(l)}$, is taken as a connection matrix. The matrix $P$ is symmetric and nilpotent one: $P^{T}=P, \quad P^{2}=P$. Besides, by definition $P \vec{\xi}^{(l)}=\vec{\xi}^{(l)}, \quad l=1,2, \ldots, p$. Consequently, the vectors $\vec{\xi}^{(l)}$ are not only the fixed points of the $P$-network, but provide the global minimum of the energy functional (5):

$$
E\left(\vec{\xi}^{(l)}\right)=-\frac{\left(P \vec{\xi}^{(l)}, \vec{\xi}^{(l)}\right)}{n}=-1, \quad l=1,2, \ldots, p .
$$

But, it is known from experience, that, as a rule, the $P$-network has additional fixed points which are called spurious fixed points. Their number is much larger that for the network with Hebb's connection matrix. And the worst is that not all the $P$-network fixed points are the local minima of the energy functional [5]. Theorem 1 helps to clarify the situation.

2). For simplicity we assume that $p$ memorized patterns $\vec{\xi}^{(l)}$ are linearly independent vectors. We introduce a rectangular $(p \times n)$ - matrix $\Xi$ whose rows are memorized patterns $\vec{\xi}^{(l)}$ :

$$
\Xi=\left(\begin{array}{cccc}
\xi_{1}^{(1)} & \xi_{2}^{(1)} & \ldots & \xi_{n}^{(1)} \\
\xi_{1}^{(2)} & \xi_{2}^{(2)} & \ldots & \xi_{n}^{(2)} \\
\ldots & \ldots & \ldots & \ldots \\
\xi_{1}^{(p)} & \xi_{2}^{(p)} & \ldots & \xi_{n}^{(p)}
\end{array}\right)
$$

Then the matrix of the orthogonal projection onto the linear subspace $\Lambda_{<\vec{\xi}^{(1)}, \vec{\xi}^{(2)}, \ldots, \vec{\xi}^{(p)}>}$ is

$$
P=Y \Xi
$$

where $Y$ is the $(n \times p)$-matrix that is pseudoinverse of the matrix $\Xi$. Apropos of the construction of pseudoinverse matrices, see [3, 田. We only want to mention, that the columns of the matrix $Y$ are $n$-dimensional vectors $\vec{y}^{(l)}$ such that $\left(\vec{y}^{(l)}, \vec{\xi}^{\left(l^{\prime}\right)}\right)=\delta_{l l^{\prime}}$, where $\delta_{l l^{\prime}}$ is the Kronecker symbol; $\vec{y}^{(l)}$ are also the linearly independent vectors.

The diagonal elements of the matrix $P$ are positive. Indeed, they are equal to the squares of the projections onto the subspace $\Lambda_{<\vec{\xi}^{(1)}, \vec{\xi}^{(2)}, \ldots, \vec{\xi}^{(p)}>}$ of the $n$-dimensional Cartesian unit vectors $\vec{e}^{(i)}=(0, \ldots, 1, \ldots, 0)$ :

$$
P_{i i}=\left(P \vec{e}^{(i)}, \vec{e}^{(i)}\right)=\left\|P \vec{e}^{(i)}\right\|^{2}=d_{i}^{2}, \quad i=1,2, \ldots, n .
$$

The values $d_{i}^{2}$ must be nonzero, otherwise the vectors $P \vec{e}^{(i)}=\sum_{j=1}^{p} \xi_{i}^{(j)} \vec{y}^{(j)}$, which are the sums of the linearly independent vectors, would be zero. Then, according to Theorem 1, the set of the $P$-network fixed points will really be wider than the set of the local minima of the energy functional.

If instead the $P$-network we consider the $P^{(0)}$-network,

$$
P^{(0)}=P-D, \text { where } D=\operatorname{diag}\left\{d_{1}^{2}, d_{2}^{2}, \ldots, d_{n}^{2}\right\}
$$


only the local minima of the energy functional will be the fixed points of the $P^{(0)}$-network. Since the memorized patterns provide the global minimum of the energy functional, they will necessarily be the $P^{(0)}$-network fixed points. In addition, the $P^{(0)}$-network has no nonphysical fixed points, i.e. those which are not the local minima of the energy functional.

Let's show that the set of the $P^{(0)}$-network fixed points has a structure which resembles the structure of the set of the fixed points for Hopfield's model [4].

\section{Theorem 3}

All $n$ neurons can be enumerated in such a way that all the $P^{(0)}$-network fixed points will be of a "piecewise constant" kind:

$$
\vec{\sigma}^{*}=(\underbrace{\varepsilon_{1}, \varepsilon_{1}, \ldots, \varepsilon_{1}}_{n_{1}}, \underbrace{\varepsilon_{2}, \varepsilon_{2}, \ldots, \varepsilon_{2}}_{n_{2}}, \ldots, \underbrace{\varepsilon_{v}, \varepsilon_{v}, \ldots, \varepsilon_{v}}_{n_{v}})
$$

where $\varepsilon_{i}=\{ \pm 1\}$. The number $v$, the composition and the dimension $n_{i}$ of constant sign intervals are defined uniquely by the memorized patterns matrix $\Xi(16)$.

The proof of Theorem 3. For simplicity we assume that the number of the neurons $n$ is much larger than the number of the memorized patterns $p$. For definiteness, we assume that $n>2^{p}$. Then it is evident that not all $n p$-dimensional column-vectors

$$
\vec{\xi}_{i}=\left(\begin{array}{c}
\xi_{i}^{(1)} \\
\xi_{i}^{(2)} \\
\vdots \\
\xi_{i}^{(p)}
\end{array}\right), \quad i=1,2, \ldots, n
$$

of the matrix $\Xi$ will be different. Some of them will be repeated 円. Let's assume that $n$ column-vectors $\vec{\xi}_{i}$ break up into $v$ groups. Each group consists of some identical vectors: $n_{1}$ identical vectors belong to the first group; $n_{2}$ identical vectors belong to the second group and so on. And $n_{1}+n_{2}+\ldots+n_{v}=n$. Without loss of generality it may be assumed that the neurons of the network can be numbered in such a way that the matrix $\Xi$ will have a form:

$$
\Xi=\left(\begin{array}{llllllllll}
\xi_{1}^{(1)} & \ldots & \xi_{1}^{(1)} & \xi_{2}^{(1)} & \ldots & \xi_{2}^{(1)} & \ldots & \xi_{v}^{(1)} & \ldots & \xi_{v}^{(1)} \\
\xi_{1}^{(2)} & \ldots & \xi_{1}^{(2)} & \xi_{2}^{(2)} & \ldots & \xi_{2}^{(2)} & \ldots & \xi_{v}^{(2)} & \ldots & \xi_{v}^{(2)} \\
\ldots & \ldots & \ldots & \ldots & \ldots & \ldots & \ldots & \ldots & \ldots & \ldots \\
\xi_{1}^{(p)} & \ldots & \xi_{1}^{(p)} & \xi_{2}^{(p)} & \ldots & \xi_{2}^{(p)} & \ldots & \xi_{v}^{(p)} & \ldots & \xi_{v}^{(p)}
\end{array}\right)
$$

It was shown in $[$ that when the projection matrix $P$ acts onto the configuration vector $\vec{\sigma}$ the result is

$$
P \vec{\sigma}=\sum_{l=1}^{p} m^{(l)}(\vec{\sigma}) \vec{\xi}^{(l)}
$$

where $m^{(l)}(\vec{\sigma})$ depends only on the state $\vec{\sigma}$. If $\vec{\sigma}^{*}$ is a fixed point of the $P^{(0)}$-network, its coordinates $\sigma^{*}$ must satisfy the system of equations:

$$
\operatorname{sign}\left(P \vec{\sigma}^{*}-D \vec{\sigma}^{*}\right) \equiv \operatorname{sign}\left(\sum_{l=1}^{p} m^{(l)}\left(\vec{\sigma}^{*}\right) \xi_{i}^{(l)}-d_{i}^{2} \sigma_{i}^{*}\right)=\sigma_{i}^{*}, \quad i=1,2, \ldots, n .
$$

\footnotetext{
${ }^{1}$ The $p$-dimentional column-vectors $\vec{\xi}_{i}(21)$ are labelled with subscripts in contrast to the $n$-dimensional memorized patterns $\vec{\xi}^{(l)}(15)$, which are labelled with superscripts.
} 
With respect to the form of the matrix $\Xi$ (see Eq.(22)), it is easy to understand that, for example, for the first $n_{1}$ values of the subscript $i$ all the sums $\sum_{l=1}^{p} m^{(l)}\left(\vec{\sigma}^{*}\right) \xi_{i}^{(l)}$ are the same: $\sum_{l=1}^{p} m^{(l)}\left(\vec{\sigma}^{*}\right) \xi_{i}^{(l)}=M, \quad i=1,2, \ldots, n_{1}$. From here it immediately follows, that the first $n_{1}$ coordinates of the fixed point $\vec{\sigma}^{*}$ must be equal. Indeed, let's contrarily assume that, for example, $\sigma_{1}^{*}=1$ and $\sigma_{2}^{*}=-1$. Then from Eqs. (23) it follows that two inequalities

$$
\left\{\begin{array}{c}
M \geq d_{1}^{2} \\
-M \geq d_{2}^{2}
\end{array}\right.
$$

have to be fulfilled simultaneously. But this is impossible.

Consequently, the first $n_{1}$ coordinates of the $P^{(0)}$-network fixed point are equal to one another. Similarly, it can be proved that the next $n_{2}$ coordinates of the fixed point are equal to one another too, and so on up to the equality among the last $n_{v}$ coordinates. The Theorem is proved.

3). Thus, the $P^{(0)}$-network possesses some attractive properties. Namely, all the memorized patterns will necessarily be its fixed points. Moreover, all the fixed points are, firstly, the local minima of the energy functional; secondly, they are of "piecewise constant" kind given by Eq. (20). It is known [4, 6] that the last two of the above mentioned properties are also typical for the fixed points of the Hopfield model, that is, for a network with the Hebb connection matrix. In our following discussions we will have in mind either the $P^{(0)}$-network, or the Hopfield model.

While Eq.(20) is only the necessary condition for a configuration to be a fixed point of a network, it restricts sufficiently the circle of the "applicants". Let's provide some estimates.

If $v$ is the number of different column-vectors of the matrix $\Xi$ and $q$ is the number of the fixed points of the related network, it is clear that $q \leq 2^{v}$. Next, when the number of the memorized patterns $p$ is given, a natural estimate for $v$ is: $v \leq 2^{p}$. But, as it was shown in [4], actually we have:

$$
q \leq 2^{2^{p-1}} .
$$

Eq. (24) restricts the number of the fixed points from above. It is easy to verify that when $p=2$, the inequality (24) transforms into the equality, and $q$ just equals 4 . But even for $p=3$ the right-hand side of Eq.(24) is 16, and in $\llbracket$ it was shown that for $p=3$ the maximum possible number of the network fixed points is 14 . This result coincides with the one of [7]. Other estimates for the fixed points number can be found in [8, 9, 10]

Partially this work was supported by RFBR grant No. 95-01-01191. 


\section{References}

[1] L.B.Litinsky. Neural network and factor analysis. Neural Network World 1996; 6:325330.

[2] E.Domany, J.L. van Hemmen and K.Schulten (Eds.). Models of neural networks. Springer-Verlag, Berlin, 1991.

[3] L.Personnaz, I.Guyon and G.Dreyfus. Collective computational properties of neural networks: new learning mechanisms. Phys. Rev. A 1986; 34: 4217-4228.

[4] L.B.Litinsky. Direct calculation of the stable points of a neural network. Theor. and Math. Phys. 1994; 101: 1492-1501.

[5] I.Kanter, H.Sompolinsky. Associative recall of memory without errors. Phys. Rev. A 1987; 35: 380-392.

[6] A.A.Vedenov, A.A.Ezhov et al.. Structure of patterns in associative memory models. In: Neural Networks - Theory and Architecture, A.V. Holden and V.I. Kryukov (Eds.), Manch. Univ. Press, pp. 169 - 186, 1991.

[7] P.Baldi. Symmetries and learning in neural network models. Phys. Rev. Lett. 1987; 59: 1976 - 1978.

[8] P.Baldi, S.S.Venkatesh. Number of stable points for sipn-glass and neural networks of high orders. Phys. Rev. Lett. 1987; 58: 913 - 916.

[9] P.Baldi. Neural networks, orientations of the hypercube, and algebraic threshold functions. IEEE Trans. on Inform. Theory 1988; 34: 523 - 530.

[10] P.Kuhlman, J.K.Anlauf. The number of metastable states in the projection rule neural network. J. Phys. A: Math., Gen. 1994; 27: 5857 - 5870. 